\title{
Adapting Interstage Home Monitoring with the use of Telemedicine During the COVID-19 Pandemic
}

\author{
Ernesto Mejia ${ }^{1,2}$ (D) Jodi Zalewski ${ }^{1,2} \cdot$ Sarah T. Plummer ${ }^{1,2}$
}

Received: 22 October 2021 / Accepted: 24 January 2022 / Published online: 22 February 2022

(c) The Author(s), under exclusive licence to Springer Science+Business Media, LLC, part of Springer Nature 2022

\begin{abstract}
Pediatric single ventricle patients have seen dramatic improvements in overall outcomes over the past several decades. This is attributed to the development of home monitoring programs for interstage patients. In today's current COVID-19 pandemic, the use of telemedicine has allowed providers to care for these patients and support their families effectively while minimizing the risk of COVID-19 exposure. Our single-center study reviewed the charts of nine patients followed by our single ventricle team through the COVID-19 pandemic. Patients discharged from the hospital and enrolled in our digital home monitoring program were included. Records were retrospectively reviewed for total number of outpatient visits, adverse events, unplanned hospital readmissions, and unplanned procedures. These results were then compared to outcomes from 2018 to 2019. In-person visits averaged every 6 weeks compared to every 2-3-week pre-pandemic. Zero adverse events reported with the use of telemedicine compared to one adverse event pre-pandemic. There was a $50 \%$ decrease in unplanned readmissions and $60 \%$ decrease in unplanned procedures during our study period. One patient was diagnosed with acute COVID-19 infection and managed conservatively via telemedicine with full recovery. To our knowledge, this is the only case-control study reporting the use of telemedicine during the COVID-19 pandemic in the interstage population. Although not statistically significant, we report a decrease in total adverse events, unplanned procedures, and unplanned admissions. Telemedicine visits allowed for identification of issues requiring hospital readmission as well as conservative management of one patient with COVID-19.
\end{abstract}

Keywords Single ventricle $\cdot$ COVID-19 $\cdot$ Telemedicine $\cdot$ In-home monitoring $\cdot$ Interstage outcomes

\section{Introduction}

Cyanotic infants, including patients with single-ventricle physiology during the interstage period are the highest risk pediatric cardiology patients. Historically prior to 2000 , the post-discharge care provided after stage 1 palliation to single ventricle patients lacked the level of surveillance needed to manage these high-risk patients safely at home, with mortality reported as high as $16 \%$ during the interstage period [1-8]. There have been dramatic improvements in the outcomes of these high-risk patients over the past 10-20 years.

Ernesto Mejia

Ernesto.Mejia@uhhospitals.org

1 University Hospitals, Rainbow Babies and Children's Hospital, 11100 Euclid Ave, Suite 380, Cleveland, OH 44106, USA

2 Division of Pediatric Cardiology, Case Western Reserve University, Cleveland, OH, USA
This is largely attributed to the development of effective and comprehensive interstage home monitoring (IHM) programs for these patients [1-8] and an AHA statement published in 2020 advocated for IHM programs as standard of care in this patient population [7].

Prior to the onset of the COVID-19 pandemic it was standard to see these patients very frequently for in-person clinic visits every 2-4 weeks in addition to their digital home monitoring which includes reporting of daily oxygen saturation, weight, and feeding volumes. Of note, echocardiograms are a necessary part of their routine follow-up and thus some in-person clinic visits are required to complete this necessary testing. However, telehealth services offer a range of options, including the daily data sharing summarized above, but also photo and video sharing and most recently video-video telemedicine visits [9].

The COVID-19 pandemic is a healthcare crisis that has required innovation to allow providers to continue to optimally care for their patients. Thankfully, pediatric patients 
with underlying structural congenital heart disease do not seem to have increased rates of poor outcomes from COVID19 , although notable cardiovascular involvement from COVID-19 has been described in both pediatric and adult literature [10]. Due to flexible regulations and increased funding during this time, there has been a specific increase in the use of face-to-face video telehealth visits which has allowed for spacing out of in-person clinic visits with reports of easy feasibility, accessibility, and positive family experience being reported by Contreras et al. [11]. There has also been documented use of telemedicine in the pediatric cardiology population with a significant increase since the beginning of the COVID-19 pandemic [12]. Prior studies have even documented the successful use of telemedicine specifically during the interstage period in high-risk cyanotic infants [9]. At our program, the use of telemedicine has allowed our clinical team to continue to follow this highrisk patient population extremely closely while mitigating the risk of COVID-19 exposure and possible infection in a patient population with significant underlying cardiac comorbidities.

At our institution, the use of video-video telemedicine visits increased significantly due to COVID-19 restrictions in our outpatient clinics and the desire to reduce exposures. Prior to COVID-19 video-video telemedicine use was overall low and was not utilized at all in our home monitoring program for high-risk cyanotic infants, with in-person visits occurring on average every 3 weeks. The aim of this case-control study was to evaluate our use of video-video telehealth visits in our high-risk infant population and its subsequent impact on outcomes in this fragile patient cohort.

\section{Methods}

After IRB approval was obtained for our single-center study, we reviewed the charts of nine patients who were followed by our single ventricle team during the early months of the COVID-19 pandemic in the USA, from March 1, 2020 to January 31,2021 . We reviewed charts for all high-risk cyanotic infants who had an initial hospital discharge and were followed in our high-risk home monitoring program and clinic. This included single ventricle interstage patients as well as two ventricle patients who underwent a palliative procedure (i.e., shunt, patent ductus arteriosus stent placement, right ventricular outflow tract stent placement, pulmonary artery band placement) and were awaiting their biventricular repair. We then reviewed the charts of nine patients followed by our team from January 1, 2018 to December 31, 2019 for comparison.

The IHM program at our institution follows the NPC-QIC and AHA recommendations [1-8]. After comprehensive education with families is completed, our high-risk cyanotic infants are discharged home with an Apple iPad (Apple Inc., Cupertino, CA) with the Locus Health monitoring platform (Locus, Charlottesville, VA) pre-loaded. Parents are also sent home with a Nellcor portable oxygen saturation monitor (Medtronic, Minneapolis, MN) and a multifunctional digital baby scale.

Parents are then asked to input daily weight, oxygen saturation, and heart rate into the Locus Health monitoring platform. In addition, feeds (including type, route, and volume), emesis, urine/stool diapers, therapies, and immunizations can be recorded. The platform also allows for video and pictures to be sent digitally to the healthcare team for further communication. The use of the iPad and Locus Health application was instituted in 2017 and has not changed since the onset of the COVID-19 pandemic. However, in an attempt to limit patient and family exposure, the use of video telemedicine visits was initiated.

The total number of outpatient visits, both in-person and via telemedicine was assessed. In addition, adverse events, unplanned hospital readmissions, and unplanned procedures were recorded. Adverse events were defined as cardiac arrest, shunt thrombosis, arrhythmia, systemic infection, aspiration, stroke, or seizures as have been previously described in the literature [1-8].

Data were analyzed for the above outcomes as well as comparing ratios between in-person to virtual visits and average number of in-person visits per month. Outcomes were compared using a Student's Paired $t$ test. The results were then compared to the outcomes reported in the literature for this high-risk population and compared to our single-center outcomes prior to the pandemic.

\section{Results}

The nine patients followed over the course of the COVID19 pandemic ranged in age from 1 to 15 months, with the median age being 3 months. Seven of the nine patients (78\%) were single ventricle patients in the interstage period. Of the patients followed pre-pandemic, eight of the nine patients (90\%) were single ventricle patients with ages ranging from newborns to 7 months, with median age of 2 months. The nine patients included in the pandemic review were followed for an average of 25.8 weeks/patient over the course of the 11-month study period. During this time, there were a total of 76 outpatient visits for the entire group (average of 8.4 visits/patient), 24 (32\%) of which were telehealth visits (Table 1). During the pandemic, there was thus one video-video telemedicine visit for every 2.8 in-person visits. Patients were still seen in-person on average every 4-6 weeks for evaluation and echocardiogram. Pre-pandemic, nine infants were followed over a 24-month period for an average of 31 weeks/patient. During this time there 
Table 1 Patient demographics

\begin{tabular}{lll}
\hline Characteristic & Pre-COVID-19 $(n=9)$ & $\begin{array}{l}\text { During } \\
\text { COVID-19 } \\
(n=9)\end{array}$ \\
\hline $\begin{array}{l}\text { Median age, mo (range) } \\
\text { Male sex, \% }\end{array}$ & $2(0.5-7)$ & $3(0.5-15)$ \\
$\begin{array}{l}\text { Diagnosis, } \% \\
\quad \text { Single ventricle }\end{array}$ & 44 & 89 \\
$\begin{array}{l}\text { Palliated future biventricular } \\
\text { repair }\end{array}$ & 11 & 78 \\
$\begin{array}{l}\text { Total outpatient visits } \\
\text { In-person, \% }\end{array}$ & 106 & 22 \\
$\quad$ Telehealth, $\%$ & $106(100)$ & 76 \\
$\begin{array}{l}\text { Median follow-up, weeks } \\
\text { (range) }\end{array}$ & $0(0)$ & $52(68)$ \\
Median visits/patient (range) & $11.8(5-20)$ & $24(32)$ \\
\hline
\end{tabular}

were 106 total outpatient clinic visits (averaged 11.8 visits/patient) and no telehealth visits. On average, there was one in-person visit performed every 6 weeks of outpatient follow-up during the pandemic period compared to one inperson clinic visit per 2-3 weeks per patient prior to the pandemic.

Our results demonstrated no adverse events across all of our high-risk cyanotic infants, including the interstage single ventricle patients during the pandemic. Although we observed a decrease in total adverse events compared to pre-pandemic, this was not found to be statistically significant $(P=0.37)$ (Fig. 1). Telemedicine visits were effective at identifying significant clinical concerns that required hospital readmission as 3 out of 9 patients required hospital admission for poor weight gain or feeding intolerance after a telemedicine visit. Patients living more than 1 hour away from the hospital reported no adverse events and the most use of telemedicine. An additional patient showed a trend of lower oxygen saturations during a telemedicine visit, prompting evaluation and hospital admission. He subsequently underwent the only unplanned procedure in our case-control study - a catheterization with recoarctation and distal Sano shunt stenosis balloon angioplasty. This is a decrease compared to total of three unplanned procedures pre-pandemic $(P=0.63)$. There were a total of 16 unplanned readmissions pre-pandemic compared to eight during the pandemic (50\% decrease), although this was again not found to be statistically significant $(P=0.17)$.

One patient had two unplanned hospital admissions for urinary tract infections, although without severe consequences and with negative blood cultures. These admissions were unrelated to either an in-person or telemedicine visit and resulted from the patient's caregiver calling with concerns regarding a cyanotic event and feeding intolerance. Only one patient had no telemedicine visits secondary to

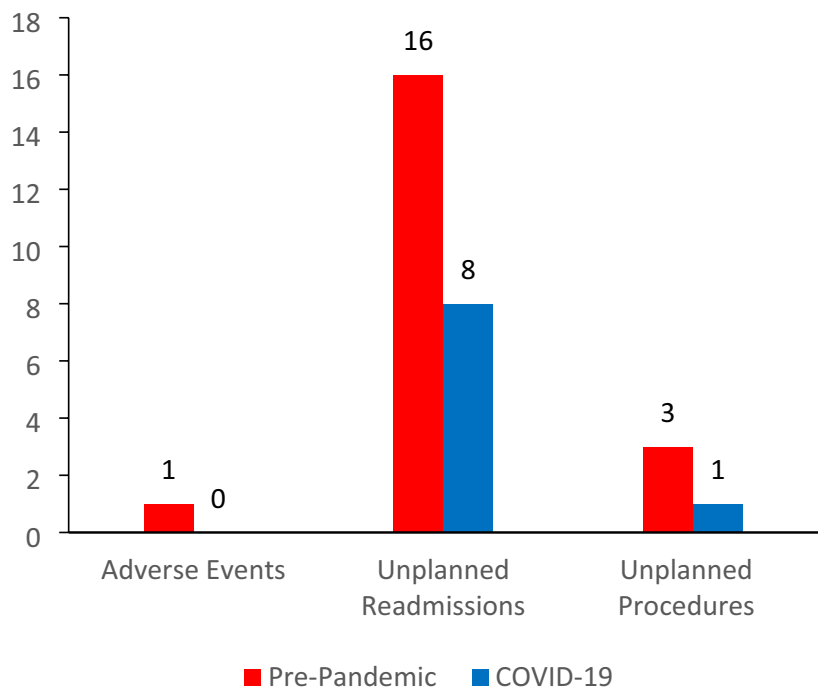

Fig. 1 Comparison of outcomes pre-pandemic (red) and during the COVID-19 pandemic (blue). No adverse events occurred during the study period with the use of telemedicine. There were also less unplanned readmissions and unplanned procedures observed with the use of telemedicine during the pandemic compared to pre-pandemic results

limited access to high-speed internet and a language barrier. This patient was followed every $2-3$ weeks in-person with interpreter services. Of note, one patient was diagnosed with COVID-19. He had mild respiratory symptoms and low-grade fever, but was able to be monitored closely and followed remotely via telemedicine. He had complete resolution of his illness without hospital admission.

\section{Discussion}

When reviewing the literature, some studies have reported improved access to patients living in rural areas due to the new widespread use of telemedicine [12]. Telemedicine has its own limitations and obstacles, including lack of standardization of telemedicine components, complex legal issues and licensure requirements, insurance reimbursements, and provider and patient acceptance [12]. Although obstacles remain, today's widespread use of smartphones worldwide and easier access to high-speed internet has provided the substrate to apply a standard framework to quickly arrange for mainstream use of patient teleconsultations [13]. The use of telemedicine was thus able to be quickly augmented with the onset of the COVID-19 pandemic and is now widespread throughout both adult and pediatric medical practice.

Although we continue to believe that patients with preexisting pulmonary and cardiovascular conditions are at higher risk for severe COVID-19 infections, it remains unclear if pediatric patients with congenital heart disease 
are truly at increased risk [14]. The literature has reported two cases of patients with congenital heart disease affected by COVID-19, both with single ventricle heart disease status post-Fontan palliation $[15,16]$. Of these two cases, one had a "severe" COVID-19 infection but was managed in the intensive care unit with conservative measures and recovered without major complications [16]. In our study, one interstage patient was diagnosed with COVID-19 infection via PCR testing after a telemedicine visit. This patient was then followed closely via frequent telemedicine visits and was managed conservatively at home through complete recovery. To our knowledge, this is the first reported case of an interstage single ventricle patient managed at home with COVID-19 with complete symptom resolution. Additionally, telemedicine visits proved effective in identifying problems with weight gain and worsening shunt/arch obstruction requiring admission and/or intervention. Telemedicine visits were thus able to prevent potential significant adverse events.

As the current global pandemic continues, we must continue to adapt and evolve the care we provide to children with congenital heart disease. While physical examination and additional testing can only be performed in-person and require this patient population to have at least a proportion of their visits in-person, the use of telemedicine is likely here to stay. This study supports the use of video-video telemedicine in this fragile patient population as a safe option to replace some percentage of in-person visits. Of note, while we found the use of video-video telemedicine visits to be effective and convenient, there have been concerns for inequities in the use telemedicine $[17,18]$. This was evident even in our patient population where one patient was not able to participate in telemedicine visits given limited access to high-speed internet/adequate phone service and a language barrier. Thus, more work is necessary to make sure that this technology can be available to all as we continue to address concerns around healthcare disparities in this fragile patient population.

\section{Conclusion}

To our knowledge, this is the only case-control study in the literature reviewing single ventricle interstage patient outcomes during the COVID-19 pandemic. Our center reports excellent outcomes in this high-risk patient population with the use of telemedicine and decreased exposure to possible COVID-19 infections related to in-person visits. In addition, we had a high-risk single ventricle patient with a confirmed symptomatic COVID-19 infection that was safely and effectively managed conservatively at home with telemedicine visits. Compared to our experience prior to the pandemic, we noted no significant difference in outcomes with the use of telemedicine in this high-risk population. Additionally, the average number of monthly in-person visits was reduced, with a concurrent decrease in total adverse events and total unplanned procedures. Thus telemedicine seems to be an effective and safe way to monitor these high-risk infants at home for at least a proportion of their visits. Thus, telemedicine will likely continue to be an integral part of the high-risk infant home monitoring program, especially as we continue to endure the effects of the COVID-19 pandemic worldwide.

Acknowledgements The authors would like to thank the sponsors, especially Richard and Wendy Halle, and all members of our IHM program, and the children and families of our study cohort.

Funding No funding was received to assist with the preparation of this manuscript.

\section{Declarations}

Conflict of interest The authors of this paper have nothing to disclose.

\section{References}

1. Oster ME et al (2015) Association of interstage home monitoring with mortality, readmissions, and weight gain. Circulation 132:502-508

2. Gardner $\mathrm{M}$ et al (2019) Association of a home monitoring program with interstage and stage 2 outcomes. J Am Heart Assoc 8:e010783

3. Castellanos DA et al (2016) Home monitoring program reduces mortality in high-risk sociodemographic single-ventricle patients. Pediatr Cardiol 37:1575-1580

4. Siehr SL et al (2014) Home monitoring program reduces interstage mortality after the modified Norwood procedure. J Thorac Cardiovasc Surg 147:718-723.e1

5. Rudd NA et al (2014) Improving interstage survival after Norwood operation: outcomes from 10 years of home monitoring. $\mathbf{J}$ Thorac Cardiovasc Surg 148:1540-1547

6. Hansen JH et al (2012) Use of surveillance criteria reduces interstage mortality after the Norwood operation for hypoplastic left heart syndrome. Eur J Cardio-thorac 41:1013-1018

7. Rudd NA et al (2020) Interstage home monitoring for infants with single ventricle heart disease: education and management. J Am Heart Assoc 9:e014548

8. Vergales J, Peregoy L, Zalewski J, Plummer ST (2020) Use of a digital monitoring platform to improve outcomes in infants with a single ventricle. World J Pediatr Congenit Hear Surg 11:753-759

9. Foster CC, Steltzer M, Snyder A et al (2020) Integrated multimodality telemedicine to enhance in-home care of infants during the interstage period. Pediatr Cardiol. https://doi.org/10.1007/ s00246-020-02489-7

10. Sabatino J, Ferrero P, Chessa M et al (2020) COVID-19 and congenital heart disease: results from a nationwide survey. J Clin Med 9(6):1774. https://doi.org/10.3390/jcm9061774

11. Contreras CM, Metzger GA, Beane JD, Dedhia PH, Ejaz A, Pawlik TM (2020) Telemedicine: patient-provider clinical engagement during the COVID-19 pandemic and beyond. J Gastrointest Surg. https://doi.org/10.1007/s11605-020-04623-5 
12. Chowdhury D, Hope KD, Arthur LC et al (2020) Telehealth for pediatric cardiology practitioners in the time of COVID19. Pediatr Cardiol 41(6):1081-1091. https://doi.org/10.1007/ s00246-020-02411-1

13. Ohannessian R, Duong TA, Odone A (2020) Global telemedicine implementation and integration within health systems to fight the COVID-19 pandemic: a call to action. JMIR Public Health Surveill 6(2):e18810. https://doi.org/10.2196/18810

14. Alsaied T, Aboulhosn JA, Cotts TB et al (2020) Coronavirus disease (COVID-19) pandemic implications in pediatric and adult congenital heart disease. J Am Heart Assoc. https://doi.org/10. 1161/jaha.120.017224

15. Ahluwalia N, Love B, Chan A, Zaidi AN (2020) COVID-19 in an adult with tricuspid atresia S/P fontan palliation. JACC 2(9):1261-1266. https://doi.org/10.1016/j.jaccas.2020.05.013

16. Linnane N, Cox DW, James A (2020) A case of COVID-19 in a patient with a univentricular heart post total cavopulmonary connection (Fontan) surgery. Cardiol Young 30(9):1350-1352. https://doi.org/10.1017/s1047951120001882

17. Zhai Y (2020) A call for addressing barriers to telemedicine: health disparities during the COVID-19 pandemic. Psychother Psychosom. https://doi.org/10.1159/000509000

18. Rajasekaran K (2020) Access to telemedicine-are we doing all that we can during the COVID-19 pandemic? Otolaryngology 163(1):104-106

Publisher's Note Springer Nature remains neutral with regard to jurisdictional claims in published maps and institutional affiliations. 